\section{Smoke and mirrors}

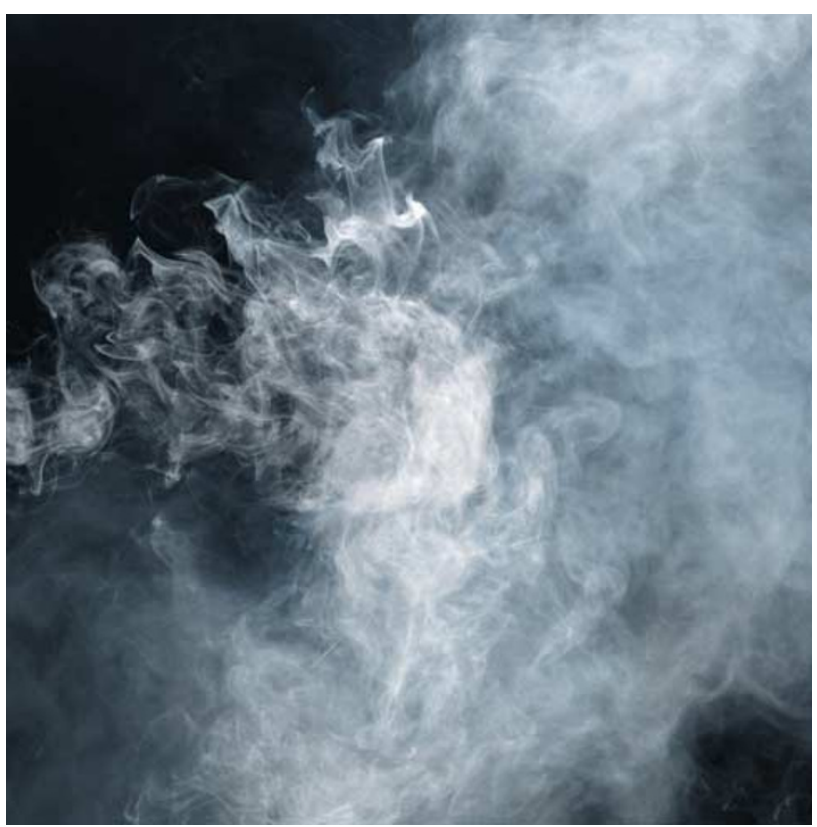

At a first glance, the statistics on the incidence of oesophageal adenocarcinoma are worrying — there has been an approximately sixfold increase in the number of cases diagnosed in the United States between 1975 and 2001. But does this reflect the actual disease burden, or is the picture warped by changes in how this cancer is detected? Heiko Pohl and H. Gilbert Welch have re-examined the data.

There were 23 cases of oesophageal adenocarcinoma diagnosed per million people in the United States in 2001. This is still rare, but it is a marked rise from 4 cases per million in 1975. As the use of endoscopy to examine patients with oesophageal complaints has also increased rapidly during this period, oesophageal cancers might be being detected that would not have produced symptoms before the patients died from other causes. Such overdiagnosis was responsible for the apparent escalation in prostate cancer seen with the introduction of screening programmes.
Furthermore, the incidence of the other major oesophageal cancer, squamous-cell carcinoma, dropped from 31 to 19 cases per million in the United States. Therefore, the seeming increase in oesophageal adenocarcinoma might represent a reclassification of cancer type due to changes in histological criteria; maybe what used to be called squamous-cell carcinoma is now more likely to be classed as adenocarcinoma.

To determine whether overdiagnosis or reclassification underlie the reported increase in oesophageal adenocarcinoma, Pohl and Welch analysed the incidence, stage and mortality statistics for oesophageal adenocarcinoma and several other cancer types, using data from the National Cancer Institute's Surveillance, Epidemiology and End Result (SEER) database. They report that overdiagnosis is unlikely to be involved, as the proportion of oesophageal adenocarcinomas that are detected in the earliest stages (in situ or localized tumours) has gone up only slightly over the period.

\section{Growing through GEFs}

Pancreatic cancer is practically incurable, with an overall 5-year survival rate of just $3 \%$. Although several signalling pathways involved in cell proliferation and apoptosis have been implicated in the development of pancreatic cancer, treatments aimed at these have proven ineffective. Martin Fernandez-Zapico and colleagues now report that VAV1, a guanine-nucleotide exchange factor (GEF) primarily expressed in bone-marrow-derived cell lineages, regulates an oncogenic pathway that promotes pancreatic cancer and could provide a new target for therapy.

The authors screened tumour samples from 95 pancreatic cancer patients and found that VAV1 was expressed in more than $50 \%$ of them, whereas it was not present in normal pancreatic tissue. Comparing the survival statistics of the patients showed that those with VAV1-positive tumours fared significantly worse than those whose tumours did not have VAV1.

Using RNA interference to deplete VAV1 in two VAV1-positive pancreatic cancer cell lines reduced the cells' ability to proliferate - compared with control cells, far fewer colonies formed in soft agar, and tumours were much smaller following injection of the cells into immunocompromised mice. Moreover, reducing the expression of VAV1 led to increased apoptosis in the VAV1positive cell lines. This was despite the fact that both cell lines also contained oncogenic KRAS, indicating that VAV1 activity somehow sustains the transformed nature of the cells.

VAV1 is one of a range of GEFs that activate members of the Rho family of GTPbinding proteins. The Rho proteins regulate several signalling cascades that lead to cell proliferation and anti-apoptotic activity, and hyperactivation of some of these proteins has been associated with cancer. To test whether the GEF function of VAV1 is important in promoting pancreatic cancer, the authors transfected DNA encoding a mutant VAV1 with no GEF activity into a VAV1-negative pancreatic cancer line. Whereas wild-type VAV1 increased proliferation of the cells, the mutant one did not.

In general, VAV1 GEF activity is modulated through cell-surface receptors, and several growth-factor receptors are aberrantly expressed in pancreatic tumours. In particular, high levels of epidermal growth factor receptor (EGFR) and its ligands are often associated with the early stages of pancreatic cancer. Fernandez-Zapico and colleagues found that addition of EGF to VAV1-expressing cells increased cellular proliferation, and showed that VAV1 rapidly becomes tyrosine phosphorylated on addition of EGF, a process that requires both the tyrosine kinase SRC and EGFR. Further investigation of the responses of the pancreatic tumour cells revealed that VAV1 is indeed involved in the regulation of an EGFstimulated pathway - the RAC1-PAK1 cascade - and that VAV1 promotes tumour proliferation at least in part through upregulating cyclin D1. The authors suggest that this oncogenic pathway, and particularly PAK1 and SRC, will be an attractive target in treating pancreatic cancer.

Helen Dell

(2) References and links ORIGINAL RESEARCH PAPER Fernandez-Zapico, M. E. et al. Ectopic expression of VAV1 reveals an unexpected role in pancreatic cancer tumorigenesis. Cancer Cell $\mathbf{7}$, 39-49 (2005) 